\title{
STIMULATION OF CRYSTALLINE LATTICE STABILIZATION OF Ga-In-P-As LAYERS DURING HETEROEPITAXY
}

\author{
J.M. OLCHOWIK \\ Department of Physics, Technical University of Lublin \\ Nadbystrzycka 38, 20-618 Lublin, Poland
}

\begin{abstract}
Every process of heteroepitaxial synthesis from liquid phase of $A^{\mathrm{III}} \mathrm{B}^{\mathrm{V}}$ compounds is based on the contact of saturated solution with the binary substrate. The initial difference in the crystalline lattice parameters or in the radii of mutually substituting atoms of the interface causes the shift of the thermodynamic balance point of the system. The change in the total energy of the system connected with this effect causes the deviation of the actual composition of the crystallising solution with respect to the planed one. In the present paper there are shown the results of the theoretical and experimental analysis of the effect of InP substrates on parameters of the $\mathrm{Ga}_{x} \operatorname{In}_{1-x} \mathrm{P}_{y} \mathrm{As}_{1-y}$ layer crystallised from liquid phase.
\end{abstract}

PACS numbers: 81.10.Dn

\section{Introduction}

The initial stage of exposure of liquid solution and binary substrate of $\mathrm{A}^{\mathrm{III}} \mathrm{B}^{\mathrm{V}}$ compounds is pseudomorphism, i.e. coherent coupling of the region separating the liquid and solid phases $[1,3]$. Due to this pseudomorphism, a change in the total energy of the system leads to a deviation in the composition of the crystallising solution from the intended one [2]. At the stage of pseudomorphism, the change in the composition of crystallising solution compared with the one which would have crystallised homogeneously is determined by the properties of the substrate and is accomplished in such a way that parameters of its crystalline lattice tend to approach these of the substrate lattice [3]. This effect is called the effect of stabilization of composition in the case of three-component solutions or stabilization of lattice constant for substituted solutions of second kind like those of $\mathrm{A}_{x} \mathrm{~B}_{1-x} \mathrm{C}_{y} \mathrm{D}_{1-y}$-type. 


\section{Theory}

The analytical model of mutual interaction of multicomponent liquid solution and binary substrate of $\mathrm{A}^{\mathrm{III}} \mathrm{B}^{\mathrm{V}}$ compounds, described in Ref. [2], enables to determine the quasi-equilibrium conditions between the elastically deformed interface and the liquid phase. Solution of a system of equations of coherent diagram of quasi-equilibrium at the interface [2], for a given temperature of contact and equilibrium liquid phase (denoted by molar indices $x$ and $y$ of its solid solution), enables to determine the composition of pseudomorphic solid phase ( $x_{\text {int }}$ and $\left.y_{\text {int }}\right)$. In the case of four-component $\mathrm{A}^{\mathrm{III}} \mathrm{B}^{\mathrm{V}}$ solutions of second kind, the stabilising action of the substrate on lattice parameters of the layers does not mean the stabilization of composition, because an isoperiodic or near isoperiodic substitution may be realised for a series of sets [4]. However, for substituted solutions of second kind, a measure of the effect of stabilization $(f)$ may be the criterion [5]:

$$
f=\frac{a_{0}-a_{\mathrm{s}}}{a_{\mathrm{coh}}-a_{0}}
$$

where $a_{0}, a_{\mathrm{s}}$ and $a_{\text {coh }}$ are the lattice constants of the equilibrium solid solution, substrate and pseudomorphic interface, respectively.

For four-component solutions, if small deviations from Vegard's law are neglected, the lattice constant of the layer in the coherent and incoherent stages of coupling with the binary substrate may be determined on the basis of equilibrium [6] and coherent equations [2] of the diagram of the system under consideration, in conjunction with the relation [4]:

$$
a_{0}=a_{24}+\left(a_{14}-a_{24}\right) x+\left(a_{23}-a_{24}\right) y+\left(a_{13}-a_{23}-a_{14}+a_{24}\right) x y,
$$

where $a_{i j}$ is the lattice constant of the binary components. This type of mathematical approach enables to analyse in a relatively easy way the effect of binary crystalline substrates of $\mathrm{A}^{\mathrm{III}} \mathrm{B}^{\mathrm{V}}$ compounds on the nature of initiation of heterogeneous nucleation of multicomponent liquid solution on the substrate surface.

\section{Results}

The results of the analysis of the effect of InP binary substrates on the parameters of $\mathrm{Ga}_{x} \mathrm{In}_{1-x} \mathrm{P}_{y} \mathrm{As}_{1-y}$ layers crystallised from the liquid phase are shown in Figs. 1 and 2. Figure 1 presents the surface of the coherent solidus of the considered heterosystem represented by molar contents of $\mathrm{Ga}\left(x_{\mathrm{int}}\right)$ and $\mathrm{P}\left(y_{\mathrm{int}}\right)$ on the interface as a function of the initial composition of the liquid solution denoted by molar indices $x$ and $y$. The experimental results from the present work are also indicated in Table.

Chemical compositions of epitaxially grown layers were determined by means of the X-ray microprobe. All the layers were crystallised by the step-cooling method with the initial supersaturation of the solution equal to $5 \mathrm{~K}$. The thicknesses of the epitaxial layers were of 1-2 $\mu \mathrm{m}$.

As seen from Fig. 1, the coherent solidus of the above heterosystems tends to approach chemical composition of layers whose lattices are matched to the substrate. A similar tendency in the dependence of the composition of this type layers on the initial composition of liquid solution was also observed in Ref. [7]. 

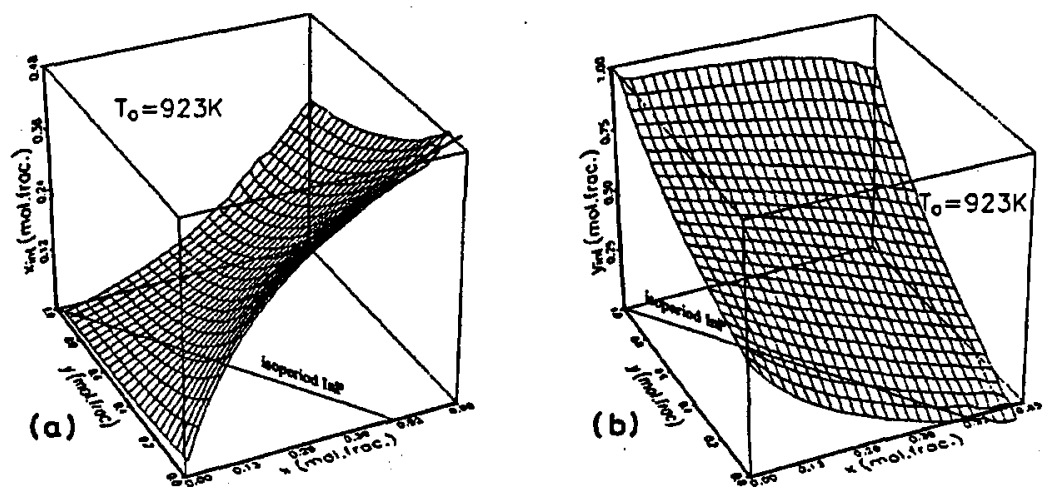

Fig. 1. Solidus surfaces of $\mathrm{Ga}_{x} \operatorname{In}_{1-x} \mathrm{P}_{y} \mathrm{As}_{1-y} / \mathrm{InP}\langle 111\rangle \mathrm{A}$ pseudomorphic heterostructure: dependence of (a) $\mathrm{Ga}$ and (b) $\mathrm{P}$ concentrations at the interface on the initial molar composition of liquid phase.

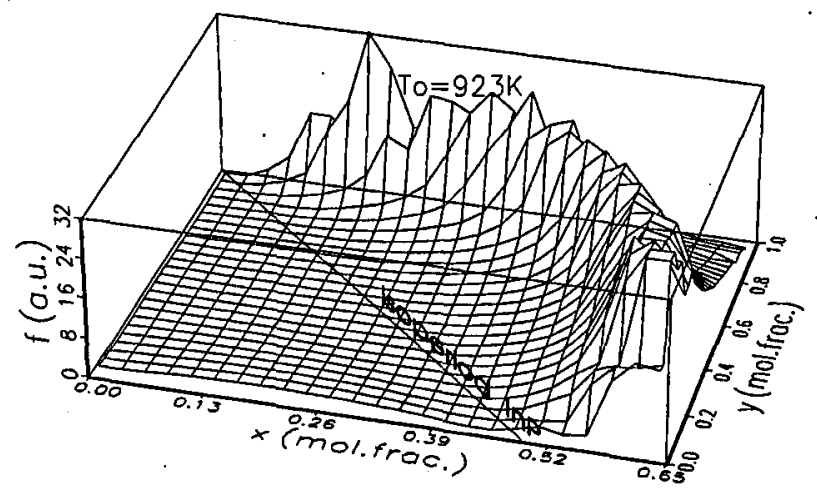

Fig. 2. Stabilization factor of lattice constants for $\mathrm{Ga}_{x} \operatorname{In}_{1-x} \mathrm{P}_{y} \mathrm{As}_{1-y}$ heterostructure and $\operatorname{InP}\langle 111\rangle$ A substrate as a function of molar composition of the initial liquid solution.

TABLE

Chemical compositions of epitaxial layers.

\begin{tabular}{c|c|c|c|c|c}
\hline $\begin{array}{c}x \\
\text { mol. frac. }\end{array}$ & $\begin{array}{c}y \\
\text { mol. frac. }\end{array}$ & $\begin{array}{c}x_{\text {int(theory) }} \\
\text { mol. frac. }\end{array}$ & $\begin{array}{c}y_{\text {int(theory) }} \\
\text { mol. frac. }\end{array}$ & $\begin{array}{c}x_{\text {int(exp.) }} \\
\text { mol. frac. }\end{array}$ & $\begin{array}{c}y_{\text {int(exp.) }} \\
\text { mol. frac. }\end{array}$ \\
\hline 0.34 & 0.01 & 0.38 & 0.06 & 0.36 & 0.07 \\
0.37 & 0.1 & 0.35 & 0.12 & 0.35 & 0.11 \\
0.49 & 0.28 & 0.34 & 0.2 & 0.36 & 0.23 \\
0.37 & 0.34 & 0.25 & 0.32 & 0.25 & 0.33 \\
0.46 & 0.37 & 0.29 & 0.28 & 0.31 & 0.32 \\
0.52 & 0.4 & 0.31 & 0.27 & 0.35 & 0.29
\end{tabular}


In the case of InP substrates, there is a large region of coherent compositions of $\mathrm{Ga}_{x} \mathrm{In}_{1-x} \mathrm{P}_{y} \mathrm{As}_{1-y}$ solutions lying near the line of isoperiodic substitution. This indicates the possibility of obtaining structures devoid of dislocations in a relatively large range of compositions, although these compositions markedly deviate from the assumed ones according to the model of equilibrium composition [6]. Obviously, the condition for the stabilising effect of the substrate on the parameters of crystalline lattice of heteroepitaxial layers is the state of pseudomorphism of the system, which is possible only below the critical thickness of the layers [8].

From Eq. (2) it is possible to determine the stabilising factor for the lattice constant for $\mathrm{Ga}_{x} \mathrm{In}_{1-x} \mathrm{P}_{y} \mathrm{As}_{1-y} / \mathrm{InP}$ system. The results of such an analysis are presented in Fig. 2. As may be seen from the figure, the stabilising effect of the substrate on the parameters of crystallised layers is most effective for compositions of initial solutions lying close to the line of isoperiodic substitution with respect to InP substrate.

Small deviation in the maximum of the stabilising factor $(f)$ of the considered system from this line results from the mass balance at the interface taken into account during calculations of the coherent diagram of the interface equilibrium, i.e.

$$
x_{i}^{l}=x_{i}^{l 0}+N_{\mathrm{s}}\left(0.5 x_{i}^{\mathrm{s}}-x_{i}^{10}\right)
$$

where $i$ denotes the component of the liquid solution, $x_{i}^{l}$ and $x_{i}^{10}$ are the concentrations in atomic parts of components in the supersaturated and saturated liquid phases, respectively, $x_{i}^{\mathrm{s}}$ is the concentration in molar parts of components in the binary substrate, and $N_{\mathrm{s}}$ is the relative number of atoms of the etched substrate.

It is known [2] that the uncompensated energy of elastic deformation of lattice in the interface region changes the energy balance of a heterosystem. In a typical heteroepitaxial process, the liquid-solid-binary substrate system is a closed one. Therefore, the condition of conservation of thermodynamic equilibrium in such situations requires the existence of a state of supersaturated solution [9]. During isothermal exposure of an initially saturated solution and a binary substrate, the latter requirement forces the necessity of excessive etching of the solid phase. Therefore, incorporating mass balance (Eq. (2)) into the set of equations makes the evaluation of effects occurring at the interface more realistic during exposure of liquid and solid phases. It follows from the fact that elastic deformation of the lattice changes the composition of the solution due to excessive etching of the substrate. In this case, the stabilising factor $(f)$ depends strongly on the initial composition of the liquid phase because the actual composition of the solution at the interface differs from the initial one during exposure [2].

\section{Conclusions}

The method of analysis of influence of the interface on the parameters of heterolayers crystallised from the liquid phase, presented in this paper, enables to determine the most appropriate starting compositions of solutions and parameters of a technological process. The main problem is only in establishing the conditions of conservation of the coherent coupling of the interface and the substrate at the stage of solid and liquid phases exposure. 


\section{References}

[1] Yu.B. Bolkhovityanov, Prog. Cryst. Growth Charact. 19, 159 (1989).

[2] J.M. Olchowik, Phys. Status Solidi A 142, 415 (1994).

[3] A.J. Finch, A.G. Quarel, Proc. R. Soc. Edinb. A 844, 398 (1933).

[4] S. Adachi, J. Appl. Phys. 58, R-1 (1985).

[5] V.V. Kuznetsov, P.P. Moskvin, V.S. Sorokin, Nonequilibrium Processes at Liquid Phase Heteroepitaxy of Semiconducting Mixed Crystals, Metallurgya, Moskva 1991 (in Russian).

[6] A.S. Jordan, M. Ilegems, J. Phys. Chem. Solids 36, 329 (1975).

[7] P.K. Bhattacharya, S. Srinivasa, J. Appl. Phys. 54, 5090 (1983).

[8] J.H. Van der Merve, J. Appl. Phys. 41, 4725 (1970).

[9] V.V. Kuznetsov, J.M. Olchowik, P.P. Moskvin, V.S. Sorokin, Inorg. Mater. 22, 1773 (1986). 